\title{
Mechanically-Prestressed Bistable Composite Laminates with Weakly Coupled Equilibrium Shapes
}

\author{
V.S.C. Chillara, M.J. Dapino* \\ Department of Mechanical and Aerospace Engineering, The Ohio State University, \\ Columbus, $\mathrm{OH}$ 43210, USA
}

\begin{abstract}
Fiber-reinforced asymmetric laminates fabricated at elevated temperatures may exhibit bistability at room temperature. The magnitude of deformation in each shape depends primarily on the curing temperature. This paper presents a novel asymmetric bistable laminate that is fabricated at room temperature and whose stable shapes are analogous to those of a fiber-reinforced laminate. The proposed laminate is composed of a stress-free isotropic core layer sandwiched between two asymmetric, mechanically-prestressed, fiberreinforced elastomeric layers. Its stable shapes can be independently tuned by varying the prestress in each elastomeric layer. The mechanics of the laminate are studied using an analytical laminated-plate model that includes the geometric and material nonlinearities associated with large deformations caused by highly-strained elastomers. The effect of core modulus, core thickness, elastomer-core width ratio, and laminate size is examined through a parametric study. Laminate samples are fabricated in the $90^{\circ} /$ core $/ 0^{\circ}$ configuration for model validation. The simulated stable shapes of the laminate are in agreement with the measured shapes. The dynamic response of the laminate during shape transition is measured using a motion capture system.
\end{abstract}

Keywords:

Laminates, Anisotropy, Internal stress, Analytical modeling, Morphing

\footnotetext{
* Corresponding author

Email addresses: chillara.1@osu.edu (V.S.C. Chillara), dapino.1@osu.edu (M.J. Dapino)
} 


\section{Introduction}

Multistable composite laminates exhibit multiple stable shapes and require actuation only for shape transition. Due to the potential offered by these laminates for reduction in weight and complexity, they are candidates for adaptive structures in automotive body panels [1], aircraft control surfaces [2], and in wind turbine blades [3]. The basic requirement in the design of a multistable laminate is to incorporate residual stress into the structure such that multiple minima in strain energy are possible. The methods available for inducing multistability in a panel can be classified into two categories, viz., mechanical and thermal.

Residual stress can be mechanically induced in isotropic panels using plastic deformation techniques like plastic forming [4], and creating dimples [5] and corrugations [6]. Forcing an initial curvature in a stress-free plate or beam by designing the appropriate boundary conditions results in pseudobistable structures $[7,8]$. Designs with mechanically-induced multistability are compatible with isotropic panels, but the resting shape is sensitive to the actuation force applied.

The most extensively studied multistable behavior involves asymmetric fiber-reinforced polymeric (FRP) laminates which are cured in a preimpregnated form at high temperature and pressure. This processing ensures geometric precision and optimal strength [9]. Cooling the cured laminates to room temperature results in an intrinsic residual stress induced by a mismatch between the thermal contraction of the matrix and fiber. At room temperature, FRP laminates exhibit two stable shapes that are curved in opposite directions [10]. The magnitude of curvature is primarily influenced by the curing temperature and its direction is governed by the orientation of fiber layers $[\mathbf{1 1}, \mathbf{1 2}, \mathbf{1 3}]$. The stable shapes of an asymmetric FRP laminate can be augmented by sandwiching an isotropic core [14]. Daynes et al. [15] selectively applied mechanical prestress to fiber layers to create a buckled region in the laminate in order to achieve symmetric curvatures. Li et al. [16] developed hybrid symmetric laminates without the need for a buckled region by including two symmetric metallic layers whose thermal expansion coefficient is much higher than that of the fibers and the matrix. In laminates with thermally induced bistability, the presence of a continuous matrix material results in fully coupled shapes at room temperature, leaving little scope for tailoring individual shapes. By virtue of the thermo-mechanical process involved in fabricating FRP laminates, they are sensitive to operat- 
ing temperature and humidity [17].

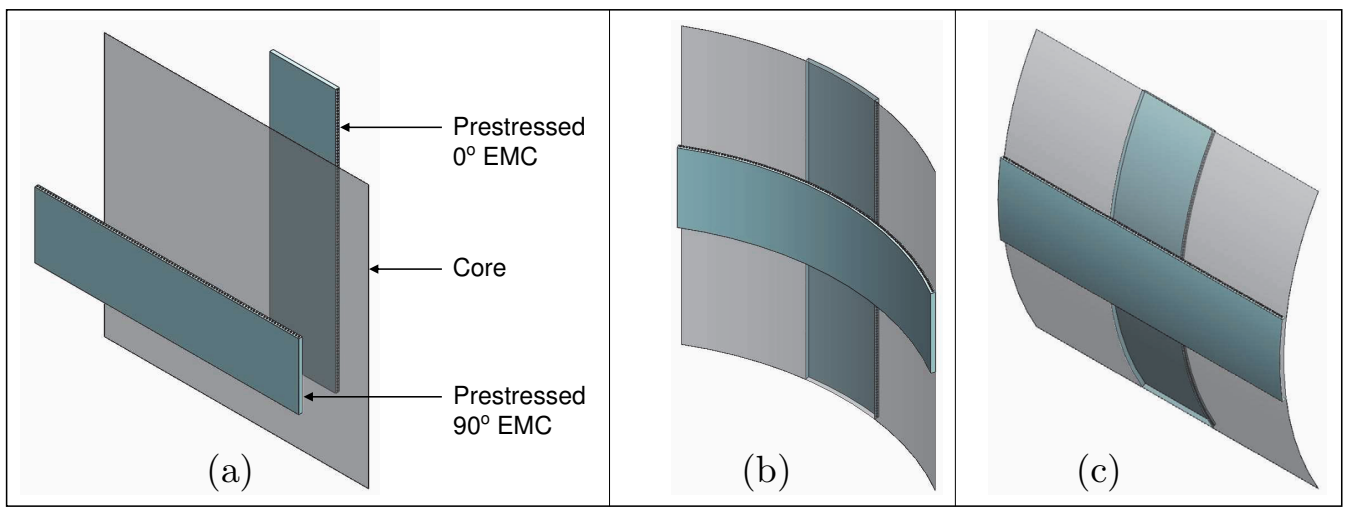

Figure 1: (a) Configuration of a mechanically-prestressed bistable laminate, (b) curved laminate due to a deformed $90^{\circ} \mathrm{EMC}$, and (c) curved laminate due to a deformed $0^{\circ}$ EMC.

This paper presents a room temperature-cured asymmetric bistable laminate with stable shapes that are analogous to those of an asymmetric FRP laminate (Figure 1). The laminate consists of a stress-free isotropic core sandwiched between two mechanically-prestressed elastomeric matrix composites (EMCs). EMCs are fiber-reinforced elastomeric layers that are intrinsically anisotropic in nature. In the proposed laminate, they are thin elastomeric strips reinforced with fibers oriented along the width in order to achieve near-zero in-plane Poisson's ratio [18]. Chillara et al. [19] demonstrated that cylindrical curvature can be created in an isotropic plate by bonding it to an EMC that is mechanically-prestressed in the matrix-dominated direction. The resulting geometry is such that the prestressed EMC is on the concave face. Two prestressed EMC strips are aligned with fibers in the $90^{\circ}$ and $0^{\circ}$ orientations and are bonded on opposite faces of the core to form a bistable laminate (Figure 1(a)). The stable cylindrical shapes in this configuration have curvatures that are $90^{\circ}$ apart (Figures $1(\mathrm{~b})$ and $1(\mathrm{c})$ ).

When the modulus of the core is much higher $\left(10^{3}\right.$ times $)$ than the modulus of an EMC in the prestressed direction, a weakly-coupled condition is possible, where the stable cylindrical shapes are independent of the prestress in the EMC on the convex face. This condition exists when the relative angle between two transversely-reinforced EMCs is $90^{\circ}$. Only the EMC on the concave face is associated with curvature since the orthogonal EMC (on the 
convex face) has near-zero in-plane Poisson's ratio. Therefore, it is possible to tune each shape independently during laminate fabrication by varying the prestress in the corresponding EMC. The magnitude of each curvature depends on the modulus and thickness of the core, and the width of each EMC. The width of an EMC is restricted to a fraction of the core width since the fibers in the EMCs can have a modulus comparable to that of the core and therefore restrict curvature in the laminate.

Varying the relative angle between the two EMCs results in complex laminate shapes consisting of twist and curvature. The proposed bistable laminate design offers opportunities for the control of motion and vibration of a continuous surface through the incorporation of localized bistability. Also, the prestressed EMCs serve as damping elements that suppress vibrations persisting after snap-through from one shape to another. Mechanicallyprestressed bistable laminates are fabricated at room temperature and their performance is expected to be insensitive to temperature and humidity variations.

A nonlinear analytical laminated-plate model inspired by the model for thermally cured bistable laminates by Hyer [20] is developed to calculate the stable shapes of a mechanically-prestressed bistable laminate (section 2). A Lagrangian strain formulation based on classical laminate plate theory is used to describe the laminate in a large deflection context. Large prestrain applied to a hyperelastic material such as an EMC requires the incorporation of material and geometric nonlinearities in the model [21]. The stable shapes of a bistable laminate are computed as a function of prestress in each of the EMCs. A fabrication method is presented and laminate samples with various magnitudes of EMC prestrain are fabricated for model validation (section 3). The calculated stable shapes of the laminate are in accordance with the measured shapes (section 4). A model-based parametric study is conducted to determine the effects of parameters such as the ratio of EMC width to core width, core modulus, core thickness, and laminate size (section $5)$. The response of the laminate during shape transition is measured using a motion capture system (section 6).

\section{Analytical Model}

The equilibrium shapes of an asymmetric mechanically-prestressed bistable laminate can be calculated analytically by minimizing its total potential energy to obtain the coefficients of the assumed strain and displacement func- 


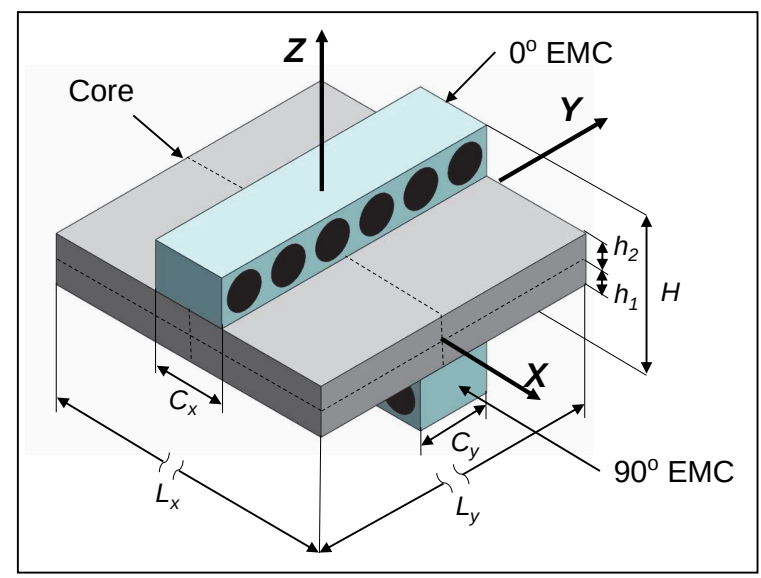

Figure 2: Schematic representation of a matrix-prestressed bistable laminate.

tions. Hyer [20] presented an analytical model based on energy minimization to calculate the room temperature shapes of thermally-cured asymmetric laminates. In-plane strain functions were assumed to be polynomials of second degree containing only the terms with an even power. Dano and Hyer [22] improved this model using third degree polynomials for strain and proposed a method for the computation of in-plane shear strain from axial strains. However, the snap-through phenomenon and certain bistability effects could not be accurately estimated using Dano's model. Pirrera et al. [23] developed higher order models to accurately reflect the geometric nonlinearity in slender laminates (high aspect ratio); the multi-event snap-through phenomenon, experimentally observed by Potter et al. [24], was explained using 11th order polynomials.

Hyer's model was successfully employed as the basis for the development of thermally-cured bistable laminate designs like adaptive [25], symmetric [15], anti-symmetric [26], and unsymmetric metal-hybrid laminates [27]. In these designs, the thermal input applied to the laminate is linearly related to strain through the coefficient of thermal expansion of the constituent layers. The model for a mechanically-prestressed bistable laminate incorporates the nonlinear mechanics of a hyperelastic material such as a prestressed fiberreinforced elastomer (Figure 2). The detailed model, based for simplicity on third order polynomials, is presented in the following subsections. 


\subsection{Laminate strain formulation}

The model for a mechanically-prestressed bistable laminate is based on the assumptions of classical laminate plate theory. Strains for composite materials with geometric nonlinearities, as applicable to this problem, are written in accordance with von Karman's hypothesis [28] as:

$$
\begin{aligned}
\epsilon_{x} & =\frac{\partial u}{\partial x}+\frac{1}{2}\left(\frac{\partial w}{\partial x}\right)^{2} \\
\gamma_{x y} & =\frac{\partial u}{\partial y}+\frac{\partial v}{\partial x}+\frac{\partial w}{\partial x} \frac{\partial w}{\partial y} \\
\epsilon_{y} & =\frac{\partial v}{\partial y}+\frac{1}{2}\left(\frac{\partial w}{\partial y}\right)^{2}
\end{aligned}
$$

Displacements $u, v$, and $w$ of any point in the composite in the $X, Y$, and $Z$ directions, respectively, are related to the displacements $u_{0}, v_{0}$, and $w_{0}$ of the geometric mid-planes (Figure 2) as:

$$
\begin{aligned}
& u(x, y, z)=u_{0}(x)-z \frac{\partial w_{0}}{\partial x}, \\
& v(x, y, z)=v_{0}(y)-z \frac{\partial w_{0}}{\partial y}, \\
& w(x, y, z)=w_{0}(x, y) .
\end{aligned}
$$

Strain of an arbitrary plane $z$ of the composite is obtained by substituting (4) - (6) into (1) - (3):

$$
\begin{aligned}
\epsilon_{x} & =\frac{\partial u_{0}}{\partial x}+\frac{1}{2}\left(\frac{\partial w_{0}}{\partial x}\right)^{2}-z\left(\frac{\partial^{2} w_{0}}{\partial x^{2}}\right) \\
\gamma_{x y} & =\frac{\partial u_{0}}{\partial y}+\frac{\partial v_{0}}{\partial x}+\frac{\partial w_{0}}{\partial x} \frac{\partial w_{0}}{\partial y}-2 z\left(\frac{\partial^{2} w_{0}}{\partial y \partial x}\right), \\
\epsilon_{y} & =\frac{\partial v_{0}}{\partial y}+\frac{1}{2}\left(\frac{\partial w_{0}}{\partial y}\right)^{2}-z\left(\frac{\partial^{2} w_{0}}{\partial y^{2}}\right)
\end{aligned}
$$

leading to the relations:

$$
\epsilon_{x}=\epsilon_{x}^{0}+z \kappa_{x}^{0}, \quad \gamma_{x y}=\gamma_{x y}^{0}+z \kappa_{x y}^{0}, \quad \epsilon_{y}=\epsilon_{y}^{0}+z \kappa_{y}^{0},
$$


where $\epsilon_{x}^{0}$ and $\epsilon_{y}^{0}$ are the in-plane axial strains, $\gamma_{x y}^{0}$ is the in-plane shear strain, and $\kappa_{x}^{0}, \kappa_{y}^{0}$, and $\kappa_{x y}^{0}$ are the curvatures and twist, respectively, of the geometric mid-plane. The displacement function $w_{0}$ in the $Z$ direction is approximated as:

$$
w_{0}(x)=\frac{1}{2}\left(a x^{2}+b x y+c y^{2}\right)
$$

such that

$$
\kappa_{x}^{0}=-\frac{\partial^{2} w_{0}}{\partial x^{2}} \triangleq-a, \quad \kappa_{x y}^{0}=-2 \frac{\partial^{2} w_{0}}{\partial y \partial x} \triangleq-b, \quad \kappa_{y}^{0}=-\frac{\partial^{2} w_{0}}{\partial y^{2}} \triangleq-c .
$$

In-plane strains are approximated by quadratic polynomials in $x$ and $y$ such that the coefficients of terms with an odd degree are zero [20]:

$$
\begin{aligned}
& \epsilon_{x}^{0}=c_{00}+c_{20} x^{2}+c_{11} x y+c_{02} y^{2}, \\
& \epsilon_{y}^{0}=d_{00}+d_{20} x^{2}+d_{11} x y+d_{02} y^{2} .
\end{aligned}
$$

Displacements $u_{0}$ and $v_{0}$, required for the calculation of shear strain, are obtained through integration of (1) and (3) as:

$$
\begin{aligned}
& u_{0}(x, y)=c_{00} x+f_{1} y+\frac{1}{2}\left(c_{11}-\frac{a b}{2}\right) x^{2} y+\left(c_{02}-\frac{b^{2}}{8}\right) x y^{2}+\frac{1}{3}\left(c_{20}-\frac{a^{2}}{2}\right) x^{3}+\frac{1}{3} f_{3} y^{3} \\
& v_{0}(x, y)=f_{1} x+d_{00} y+\frac{1}{2}\left(d_{11}-\frac{c b}{2}\right) x y^{2}+\left(d_{20}-\frac{b^{2}}{8}\right) x^{2} y+\frac{1}{3}\left(d_{02}-\frac{c^{2}}{2}\right) y^{3}+\frac{1}{3} f_{2} x^{3}
\end{aligned}
$$

Substitution of (11), (15), and (16) in (8) yields an expression for shear strain in the composite.

\subsection{Nonlinear model of a prestressed EMC}

A large prestrain (up to 1.0) in the matrix-dominated direction of an EMC is associated with a geometric nonlinearity and a hyperelastic material response [21]. The novelty in the proposed model is in the incorporation of these nonlinearities into the basic analytical framework for calculating the stable shapes of bistable laminates [20]. The fibers in the EMC strip are assumed to be oriented along its width in order to maintain a near-zero inplane Poisson's ratio. There is no loss of generality in this assumption since the relative angle between the two EMCs is sufficient to design the orientations of the principal curvatures in the laminate. Decoupling the lateral and 
longitudinal strains in an EMC allows the designer to use a unidirectional polynomial stress function instead of a hyperelastic model, thereby reducing computational cost. A model for a prestressed EMC that is applied as a lamina in a morphing composite has been presented by Chillara et. al [29] and is outlined here for completeness. The nonlinear Lagrangian strain $\left(e_{x}^{(90)}\right)$ of

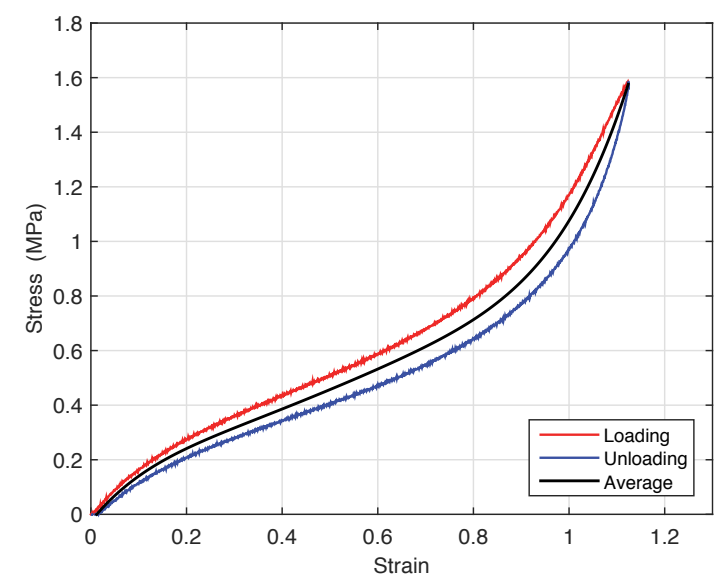

Figure 3: Stress-strain curve for a $90^{\circ}$ EMC obtained from a uniaxial tensile test.

a $90^{\circ}$ EMC prestressed in the $X$ direction is written as:

$$
e_{x}^{(90)}=\frac{\partial u}{\partial x}+\frac{1}{2}\left(\frac{\partial u}{\partial x}\right)^{2}
$$

where $u$ is the axial displacement. For compatibility with (1), this strain must be linearized. The corresponding linear axial strain is:

$$
\varepsilon_{x}^{(90)}=\frac{\partial u}{\partial x}
$$

The linear strain $\varepsilon_{x}^{(90)}$ is expressed in terms of $e_{x}^{(90)}$ as:

$$
\varepsilon_{x}^{(90)}=-1+\sqrt{1+2 e_{x}^{(90)}} .
$$

Stress is calculated incrementally at discrete values of linear strain as:

$$
\sigma_{i}=\sigma_{i-1}+E_{i}\left(\varepsilon_{i}-\varepsilon_{i-1}\right)
$$


where $E_{i}$ is the point-wise modulus obtained from a uniaxial tensile test of a $90^{\circ} \mathrm{EMC}$ (Figure 3). The design details of the tested $90^{\circ} \mathrm{EMC}$ are presented in section 3.

The reduced average stress function for an EMC with near-zero in-plane Poisson's ratio and with fibers oriented at $90^{\circ}$ in the bistable laminate, is described using a quartic polynomial as:

$$
\sigma_{x}=-0.698 \varepsilon_{x}^{4}+2.29 \varepsilon_{x}^{3}-2.306 \varepsilon_{x}^{2}+1.598 \varepsilon_{x} \quad[\mathrm{MPa}] .
$$

The stress function $\left(\sigma_{y}\right)$ for the corresponding $0^{\circ} \mathrm{EMC}$ in the laminate is obtained by replacing $\varepsilon_{x}$ in (21) with $\varepsilon_{y}$. The stress function for an EMC in an arbitrary orientation can be obtained using the appropriate rotation matrices.

\subsection{Computation of stable laminate shapes}

The potential energy of the system $\left(U_{T}\right)$ can be expressed as a function of the geometric and material properties of the laminae, total strains of the laminate, and prestrain in the EMCs as:

$$
U_{T}=\int_{V}\left(U_{1}+Q_{12} \epsilon_{x} \epsilon_{y}+U_{2}+\frac{1}{2} Q_{16} \gamma_{x y} \epsilon_{x}+\frac{1}{2} Q_{26} \gamma_{x y} \epsilon_{y}+\frac{1}{2} Q_{66} \gamma_{x y}^{2}\right) \mathrm{d} V
$$

where $\left\{Q_{i j}\{i, j=1,2,6\}\right\}$ are the plane stress-reduced stiffness parameters [28], and $U_{1}=0.5\left(Q_{11} \epsilon_{x}^{2}\right), U_{2}=0.5\left(Q_{22} \epsilon_{y}^{2}\right)$ are the strain energies in the linearly strained directions in a lamina. Energies $U_{1}$ and $U_{2}$ are computed as the integral of $\sigma_{x}$ and $\sigma_{y}$ for a $90^{\circ}$ and $0^{\circ}$ EMC respectively. This gives, for the two EMCs:

$$
U_{1}^{(90)}=f\left(\epsilon_{90}-\epsilon_{x}\right), \quad U_{2}^{(0)}=f\left(\epsilon_{0}-\epsilon_{y}\right)
$$

where $\epsilon_{90}$ and $\epsilon_{0}$ are the prestrains in the $90^{\circ}$ and the $0^{\circ}$ EMCs respectively. The limits of integration for the computation of strain energy are listed in Table 1.

The equilibrium shapes of the laminate are obtained by minimizing $U_{T}$ using the following variational approach:

$$
\delta U_{T}=\sum_{i=1}^{14} \frac{\partial U_{T}}{\partial c_{i}}=0
$$


Table 1: Limits of integration for the computation of the total potential energy of a mechanically-prestressed bistable laminate.

\begin{tabular}{lccc}
\hline Lamina & $x$ & $y$ & $z$ \\
\hline $90^{\circ}$ EMC & $\left(-L_{x} / 2, L_{x} / 2\right)$ & $\left(-C_{y} / 2, C_{y} / 2\right)$ & $\left(-H / 2,-h_{1}\right)$ \\
Core & $\left(-L_{x} / 2, L_{x} / 2\right)$ & $\left(-L_{y} / 2, L_{y} / 2\right)$ & $\left(-h_{1}, h_{2}\right)$ \\
$0^{\circ}$ EMC & $\left(-C_{x} / 2, C_{x} / 2\right)$ & $\left(-L_{y} / 2, L_{y} / 2\right)$ & $\left(h_{2}, H / 2\right)$ \\
\hline
\end{tabular}

where,

$$
c_{i}=\left\{a, b, c, c_{00}, c_{20}, c_{11}, c_{02}, d_{00}, d_{20}, d_{11}, d_{02}, f_{1}, f_{2}, f_{3}\right\}
$$

The fourteen equations resulting from (24) are solved simultaneously to calculate the strains and the out-of-plane displacement of the laminate. The expressions for $U_{T}$ and $\delta U_{T}$ are evaluated in symbolic form using MAPLE. The Newton-Raphson approach is employed to numerically approximate the equilibrium shapes of the laminate. Due to the sensitivity of the nonlinear solver to initial conditions, the initial estimates for the simulation are chosen based on the measured shapes of one fabricated sample. To obtain solutions corresponding to the stable shapes, a constraint on the Jacobian of the system of equations is included in the model. The Jacobian matrix is computed with respect to the variables listed in (25) and is required to be positive definite in order to have a stable solution.

\section{Laminate Fabrication}

A fabrication procedure for a mechanically-prestressed bistable laminate is presented in this section. Samples with different values of EMC prestrain are fabricated in a $90^{\circ} \mathrm{EMC} / \mathrm{spring}$ steel $/ 0^{\circ} \mathrm{EMC}$ configuration. The two stable shapes of the resulting laminate are measured using a motion-capture camera system.

\subsection{Elastomeric matrix composite}

Two layers of unidirectional carbon fibers are wetted with liquid silicone and then sandwiched between two pre-cured silicone rubber strips to create an elastomeric matrix composite. The unidirectional fibers are oriented along 
the width of the EMC strip. Rhodorsil V340/CA45 mold making silicone rubber of durometer grade 45 (shore A) is used to prepare pre-cured sheets of $0.76 \mathrm{~mm}$ thickness. Unidirectional carbon fibers are prepared from a woven fabric that has an areal density of $3.1 \mathrm{~kg} / \mathrm{m}^{2}$ (Fiberglast Developments Corp.) The dimensions of the fabricated EMCs are $152.4 \times 38.1 \times 2 \mathrm{~mm}$ and the volume fraction of the fibers is 0.17 .

The longitudinal $\left(E_{1}\right)$ and transverse $\left(E_{2}\right)$ modulus of an EMC that is reinforced along its width can be calculated using the rule of mixtures as:

$$
E_{1}=\frac{1}{\frac{\nu_{f}}{E_{f}}+\frac{1-\nu_{f}}{E_{m}}}, \quad E_{2}=E_{f} \nu_{f}+E_{m}\left(1-\nu_{f}\right) .
$$

For a fiber modulus of $240 \mathrm{GPa}$ and a linear matrix modulus of $1.2 \mathrm{MPa}$ (up to 0.2 strain), $E_{1}$ and $E_{2}$ are calculated to be $1.45 \mathrm{MPa}$ and $40.8 \mathrm{GPa}$, respectively. $E_{1}$ is in agreement with the measured longitudinal modulus of $1.5 \mathrm{MPa}$ in the linear regime (Figure 3 ). However, $E_{2}$ may not reflect the true mechanics of the EMC in a laminated-composite setup, since the fibers are not uniformly distributed within the matrix.

\subsection{Bistable laminate}

The core layer is a sheet of spring steel of dimensions $152.4 \times 152.4 \times$ $0.127 \mathrm{~mm}$. A paint primer (Rust-Oleum) is sprayed on the sheet to create a rough surface for bonding the EMC to steel; interlaminar shear, which is a common failure mode in prestressed composites, is improved $[30,31]$. One of the fabricated EMCs is stretched using a pair of grips and is then bonded to the steel sheet such that the fiber orientation in the laminate is $90^{\circ}$ (Figure $4(\mathrm{a})$ ). The sample is allowed to cure for 24 hours during which pressure is applied to the bonded region throughout this duration (Figure 4(b)). The resulting laminate has a cylindrical shape at equilibrium (Figure $4(\mathrm{c}))$. This laminate is then bonded to a prestrained EMC such that the fibers are in a $0^{\circ}$ orientation (Figure $4(\mathrm{~d})$ ). The sample is flattened using two thick plates and is held down using clamps for curing (Figure 4(e)). Flattening the sample ensures minimal in-plane strain in the steel sheet when the $0^{\circ}$ EMC is bonded to it. Fabrication trials revealed that debonding can occur during handling at the EMC-core interface at the edges of the laminate. This phenomenon is attributed to a sharp transition in stress state from the prestressed region in the EMC to the stress-free region in the core. Debonding can be prevented by curling and bonding the dangling ends of the EMC onto the opposite face of the sheet (Figure 4(f)). 


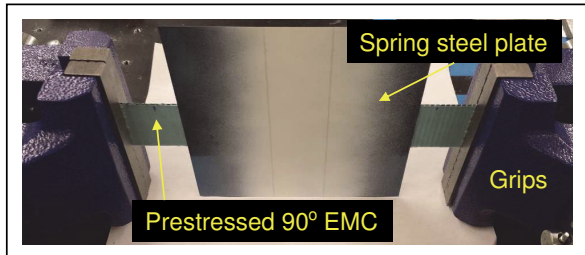

(a)

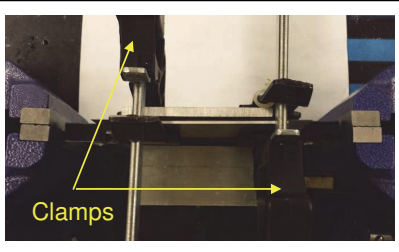

(b)

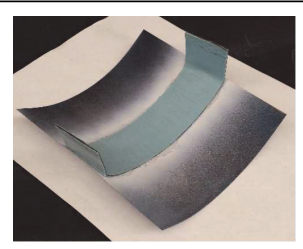

(c)

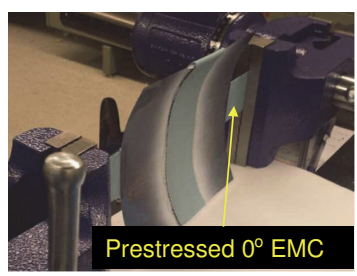

(d)

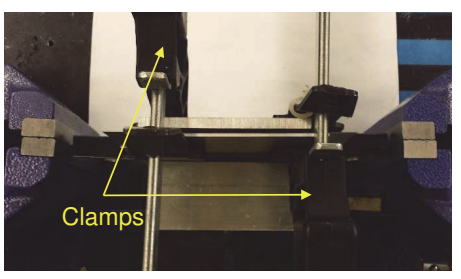

(e)

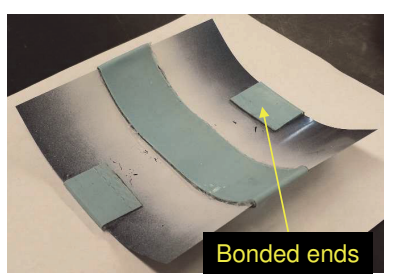

(f)

Figure 4: (a) A spring-steel core bonded to a prestressed EMC in the $90^{\circ}$ orientation, (b) pressure applied to the bonded region for curing, (c) laminate with a single curvature obtained upon removal of the EMC from the grips, (d) curved sample bonded to an EMC in the $0^{\circ}$ orientation, (e) pressure applied to the bonded region after flattening the sample, (f) resulting bistable laminate with the ends of the EMCs wrapped around and bonded to the core.

The fabricated bistable laminate sample has two stable shapes as shown in Figure 5. Curvature in the laminate due to the $90^{\circ}$ EMC (Figure 4(c)) is found to remain unaltered after bonding the $0^{\circ}$ EMC to it (Figure 5(a)). However, the region spanning the width of the $0^{\circ}$ EMC has a flattened appearance. This is due to the presence of two layers with very high modulus, viz., the carbon fibers in the EMC and the steel core in this region. Transition between the stable shapes is achieved by applying a moment at the ends of the composite about the axis of initial curvature.

\subsection{Measurement of laminate geometry}

The cylindrical stable shapes of the laminate are recorded using a 3D image capture technique. The process involves the reconstruction of a surface by mapping the coordinates of markers physically attached to the surface [32]. The image of the surface is captured using multiple cameras and the coordinates of the markers are obtained by triangulating the marker locations captured by each camera. The shapes of the fabricated laminate are measured using an OptiTrack (NaturalPoint Inc.) motion capture system that 


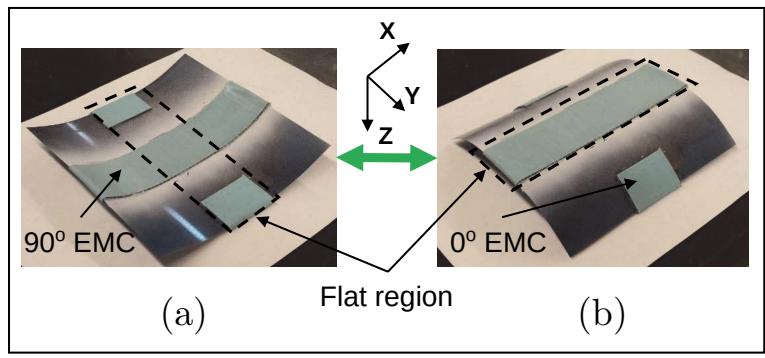

Figure 5: Stable shapes of a fabricated sample of a mechanically-prestressed bistable laminate.

maps the coordinates of 49 hemispherical reflective markers. Each marker has a diameter of $3 \mathrm{~mm}$ and is bonded to the sample in a $7 \times 7$ grid (Figure $6(\mathrm{a}))$. The horizontal and vertical distances between markers is $25.4 \mathrm{~mm}$ (1 inch). Four still cameras with a resolution of 1.3 megapixels and a maximum recording speed of 120 frames per second are arranged as shown in Figure 6(b). Using the Motive (NaturalPoint Inc.) software, it is ensured that each marker is seen at least by 3 cameras. The cameras are calibrated

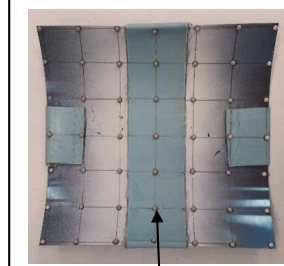

Reflective markers

(a)

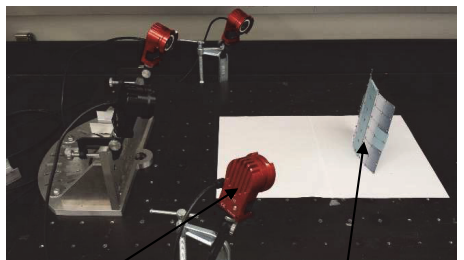

Cameras (4) Composite

(b)

Figure 6: (a) Reflective markers bonded to the laminate sample for shape measurement, (b) setup showing the motion capture system used to record the equilibrium shapes of the laminate.

to an accuracy of $0.03 \mathrm{~mm}$ in a capture volume of $305 \times 305 \times 305 \mathrm{~mm}$ by waving a wand that contains three spherical reflective markers located inline at a fixed relative distance. A coordinate system is defined using a right angle measure that is equipped with spherical reflective markers at its ends. Two axes are obtained from these markers while the third axis is calculated as their cross product. The laminate sample is then placed in the capture 
volume in each of its stable shapes and the coordinates of each marker are obtained. The recorded marker coordinates are normalized and are fit using a quartic-quadratic polynomial to reconstruct each cylindrical shape of the laminate. While a quadratic-linear polynomial is sufficient to describe a cylindrical shape, a quartic-quadratic polynomial is required to capture the flat region in the laminate enforced by the EMC on the convex face. The reconstructed stable shapes of the laminate are plotted in Figures 7 and 8.

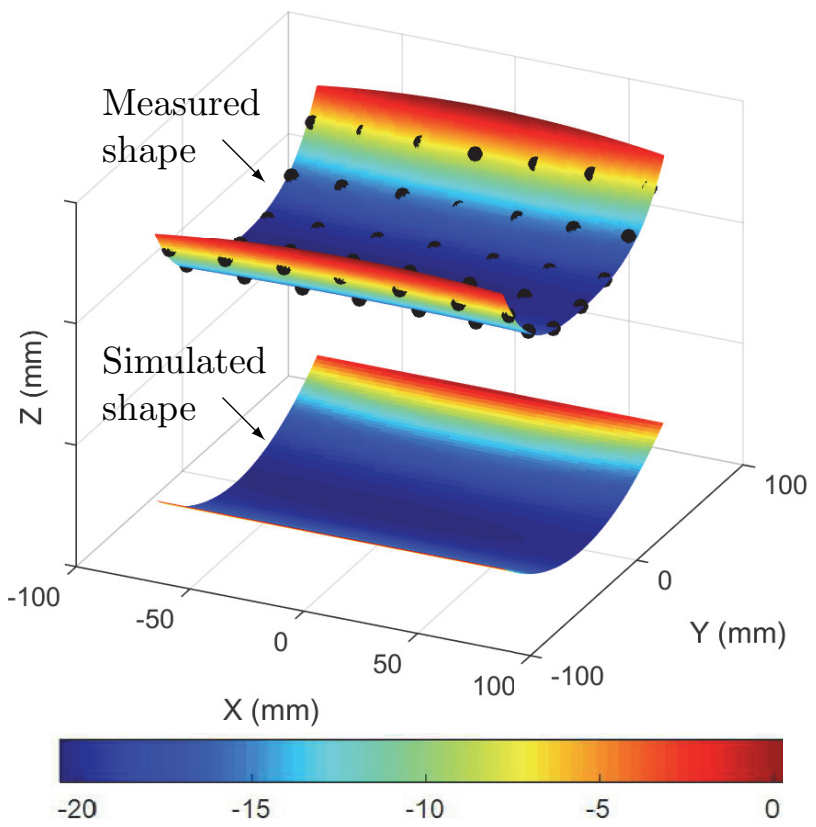

Figure 7: Stable cylindrical shape of a mechanically-prestressed bistable laminate with curvature about the $X$ axis.

\section{Results and Discussion}

The simulations conducted using the model presented in section 3 yield three equilibrium shapes for a mechanically-prestressed bistable laminate. Two of these shapes are stable and have a cylindrical geometry while one shape is unstable and has a saddle geometry. The simulated shapes are as expected since the fabricated laminate exhibits only two cylindrical shapes.

The material properties and dimensions of the laminae used in the simulation are listed in Tables 2 and 3 respectively. The in-plane shear modulus 


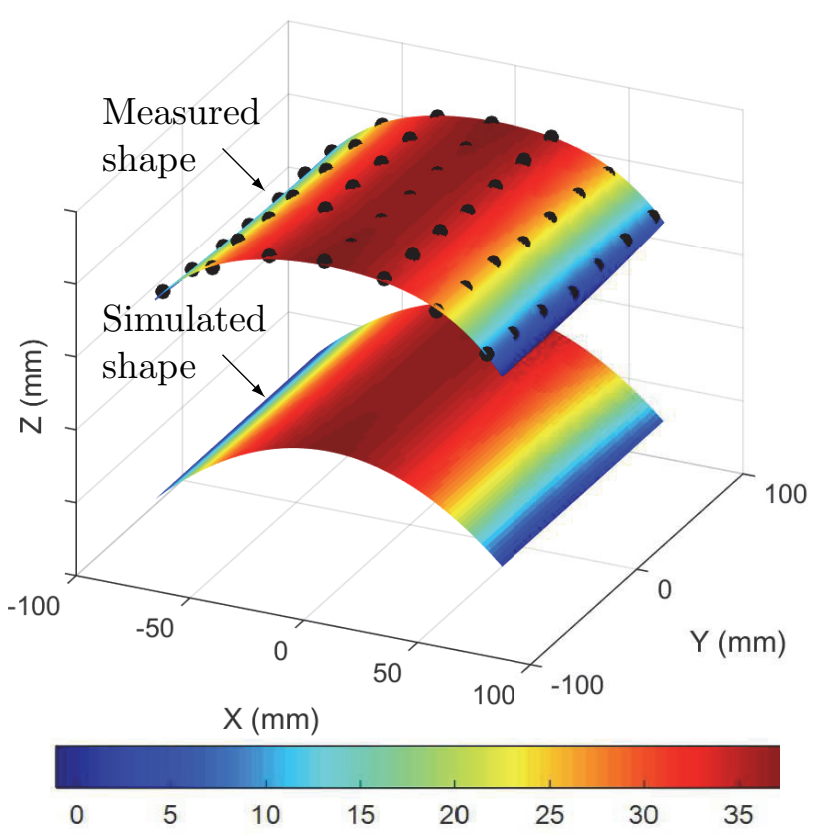

Figure 8: Stable cylindrical shape of a mechanically-prestressed bistable laminate with curvature about the $Y$ axis.

$\left(G_{12}\right)$ for a $90^{\circ}$ and a $0^{\circ} \mathrm{EMC}$ is assumed to be 0.8 times its linear elastic modulus in the matrix-dominated direction [33]; $G_{12}$ for an EMC is a constant since no twist is expected in the stable shapes of a laminate in a $90^{\circ} / \mathrm{core} / 0^{\circ}$ configuration. Poisson's ratios $\nu_{12}$ and $\nu_{21}$ for an EMC are assumed to be zero due to the high ratio of transverse (40.8 GPa) to longitudinal $(1.5 \mathrm{MPa})$ modulus in the linear regime.

Simulations conducted with a transverse EMC modulus of $40.8 \mathrm{GPa}$ (see section 3.1) result in very small curvatures on the order of $10^{-5} \mathrm{~mm}^{-1}$. These calculations are consistent with the flat regions shown in Figure 5(b). However, global curvature is calculated by using the same curvature for both flat and curved regions. The assumption of constant curvature is implemented by setting the transverse EMC modulus to $0.4 \mathrm{MPa}$. This assumption is justified because the modified modulus value corresponds to shear in the purely-elastomeric sub-layer that is located between the core and the fiberreinforced elastomeric layer in the EMC; the restriction offered by the fibers in the EMC is nullified.

For a prestrain of 0.6 in each of the EMCs, the stable shapes calculated 
Table 2: Material properties of the laminae for modeling and fabrication.

\begin{tabular}{lrrrrr}
\hline Lamina & $E_{1}(\mathrm{MPa})$ & $E_{2}(\mathrm{MPa})$ & $G_{12}(\mathrm{MPa})$ & $\nu_{12}$ & $\nu_{21}$ \\
\hline $90^{\circ}$ EMC & Nonlinear & 0.4 & 1.2 & 0 & 0 \\
Core layer & 200,000 & 200,000 & 78,125 & 0.28 & 0.28 \\
$0^{\circ}$ EMC & 0.4 & Nonlinear & 1.2 & 0 & 0 \\
\hline
\end{tabular}

Table 3: Dimensions of the laminae for modeling and fabrication

\begin{tabular}{lccc}
\hline Lamina & Length $(\mathrm{mm})$ & Width $(\mathrm{mm})$ & Thickness $(\mathrm{mm})$ \\
\hline $90^{\circ}$ EMC & 6.0 & 1.5 & 0.080 \\
Core & 6.0 & 6.0 & 0.005 \\
$0^{\circ}$ EMC & 1.5 & 6.0 & 0.080 \\
\hline
\end{tabular}

with the updated EMC modulus are compared with the corresponding measured shapes (Figures 7 and 8). The principal curvatures $\kappa_{x}^{0}$ and $\kappa_{y}^{0}$ of the fabricated sample are calculated using the method of least squares as -0.0093 $\mathrm{mm}^{-1}$ and $0.0057 \mathrm{~mm}^{-1}$ respectively. It is observed that $\left|\kappa_{x}^{0}\right|$ is greater than $\left|\kappa_{y}^{0}\right|$; the cause is a residual in-plane strain in the core when it is flattened out for bonding with the $0^{\circ}$ EMC (Figure $4(\mathrm{e})$ ). However, the average of $\left\{\left|\kappa_{x}^{0}\right|,\left|\kappa_{y}^{0}\right|\right\}$ of $0.0071 \mathrm{~mm}^{-1}$ closely matches the simulated $\left\{\left|\kappa_{x}^{0}\right|\right.$ and $\left.\left|\kappa_{y}^{0}\right|\right\}$ of $0.0076 \mathrm{~mm}^{-1}$.

The stable cylindrical shapes of a laminate in the $90^{\circ} \mathrm{EMC} / \mathrm{core} / 0^{\circ} \mathrm{EMC}$ configuration are simulated as a function of the EMC prestrains $\epsilon_{90}$ and $\epsilon_{0}$ (Figure 9). The associated principal curvatures $\kappa_{x}^{0}$ and $\kappa_{y}^{0}$ have a nonlinear dependence on $\epsilon_{90}$ and $\epsilon_{0}$ respectively. The nonlinear variation bears resemblence to the hyperelastic response of an EMC (Figure 3). Curvatures $\kappa_{x}^{0}$ and $\kappa_{y}^{0}$ are respectively independent of $\epsilon_{0}$ and $\epsilon_{90}$. Since the EMCs are prestressed by applying a corresponding strain, a weakly-coupled condition exists such that each curvature is independent of the prestress in the EMC on the convex face. This condition is valid only if the prestressed EMCs are orthogonally oriented on an isotropic core; there is no twist in the laminate.

Seven laminate samples with prestrain values $\left(\epsilon_{90}, \epsilon_{0}\right)$ of $(0.3,0.3),(0.4,0.4)$, $(0.5,0.5),(0.6,0.6),(0.8,0.8),(0.3,0.6)$, and $(0.4,0.8)$ are fabricated and their curvatures are measured (Figure 9). The simulated curvatures of the laminate are in agreement with the measured curvatures. The weakly-coupled 


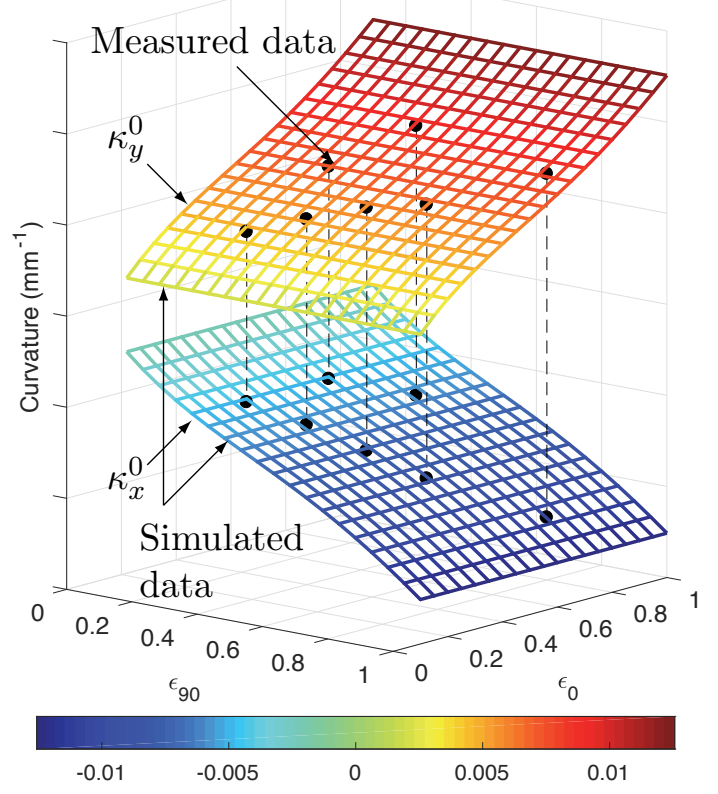

Figure 9: Equilibrium curvatures of a mechanically-prestressed bistable laminate as a function of prestrain in the $90^{\circ}$ and $0^{\circ}$ EMCs.

condition is validated by the fact that $\kappa_{y}^{0}$ is equal in samples with equal $\epsilon_{0}$ but unequal $\epsilon_{90}$. The bias between $\left|\kappa_{x}^{0}\right|$ and $\left|\kappa_{y}^{0}\right|$ induced in the fabrication process is found to be constant in all samples. Accuracy of the designed shapes can be improved through simultaneous lamination but at the expense of increased complexity of the fabrication setup.

\section{Parametric Study}

A study on the effect of design parameters such as core modulus, core thickness, and size on laminate shapes is presented in this section. It is shown that besides EMC prestrain, laminate shapes can be tailored using the ratio of EMC width to core width. Material and geometric properties listed in Tables 2 and 3 are used in the analyses unless mentioned otherwise.

\subsection{Effect of EMC width}

A change in the width of an EMC in the laminate is associated with a change in the magnitude of prestress developed in it and hence affects 


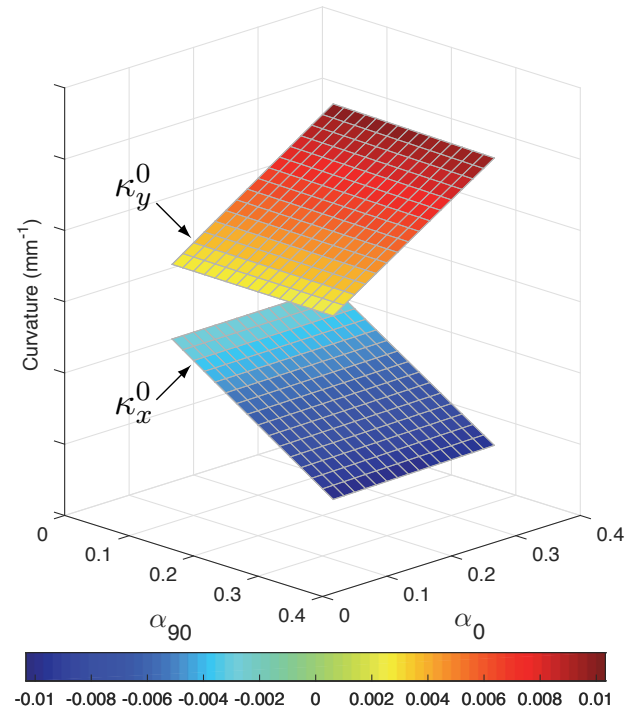

Figure 10: Influence of the width of an EMC relative to core width on the stableequilibrium curvatures of the laminate. $\epsilon_{90}=\epsilon_{0}=0.6$.

laminate geometry. The non-dimensional widths of the EMCs relative to the core dimensions are defined as:

$$
\alpha_{90}=\frac{C_{y}}{L_{y}}, \quad \alpha_{0}=\frac{C_{x}}{L_{x}} .
$$

The stable curvatures for $\alpha_{90}$ and $\alpha_{0}$ ranging from 0.08 to 0.33 are plotted in Figure 10. Prestrains $\epsilon_{90}$ and $\epsilon_{0}$ are maintained constant at 0.6. It is seen that $\kappa_{x}^{0}$ and $\kappa_{y}^{0}$ are a linear function of $\alpha_{90}$ and $\alpha_{0}$ respectively. Such a trend can be attributed to a linear relationship between the width and the cross-sectional area, and hence the prestress, of an EMC. Further, $\kappa_{x}^{0}$ and $\kappa_{y}^{0}$ are independent of $\alpha_{0}$ and $\alpha_{90}$ respectively, indicating the weak coupling between the EMCs.

\subsection{Effect of core modulus and thickness}

The modulus $(E)$ and thickness $\left(t=h_{2}-h_{1}\right)$ of the isotropic core affect the two stable shapes of the laminate. Curvatures $\kappa_{x}^{0}$ and $\kappa_{y}^{0}$ are calculated for $\epsilon_{90}=0.8$ and $\epsilon_{0}=0.5$ over a range of core modulus and thickness. Figure 11 is a plot of the isometric lines of $-1 /\left|\kappa_{x}^{0}\right|$ and $-1 /\left|\kappa_{y}^{0}\right|$. For a given $t,\left|\kappa_{x}^{0}\right|$ and $\left|\kappa_{y}^{0}\right|$ decrease with an increase in $E$. Further, $\left|\kappa_{x}^{0}\right|$ and $\left|\kappa_{y}^{0}\right|$ decrease with 


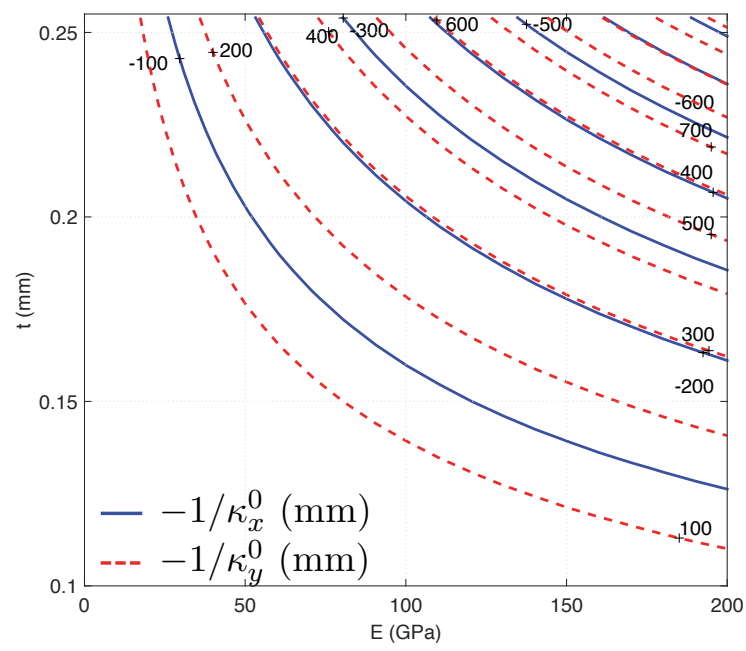

Figure 11: Stable-equilibrium curvatures of the laminate as a function of core modulus and thickness. $\epsilon_{90}=0.8$ and $\epsilon_{0}=0.5$.

an increase in $t$ for a fixed $E$. Figure 11 serves as a tool for the selection of the thickness of the chosen core material to obtain a given set of laminate curvatures. For example, a core material of modulus $60 \mathrm{GPa}$ must be 0.185 $\mathrm{mm}$ thick such that the stable shapes of the laminate have radii of curvature $\left(\left|1 / \kappa_{x}^{0}\right|\right.$ and $\left.\left|1 / \kappa_{y}^{0}\right|\right)$ of $100 \mathrm{~mm}$ and $150 \mathrm{~mm}$.

\subsection{Effect of laminate size}

In thermally-cured thin unsymmetric laminates, bistability is observed beyond a particular laminate size [20]. To examine a relevant feature, the bistability of a mechanically-prestressed square laminate is simulated for characteristic length $L\left(=L_{x}=L_{y}\right)$ ranging from $25.4 \mathrm{~mm}$ to $228.6 \mathrm{~mm}$. EMC prestrains $\epsilon_{90}$ and $\epsilon_{0}$ are held constant at 0.6. Figure 12 shows the variation of $\kappa_{x}^{0}$ and $\kappa_{y}^{0}$ with $L$. It is seen that the laminate has a single stable shape up to a bifurcation length $\left(L_{b}\right)$ beyond which it has two stable shapes. The solid and dotted lines represent the major and minor curvatures respectively, for a given stable shape. Each shape beyond $L_{b}$ is non-cylindrical up to a critical characteristic length $\left(L_{c}\right)$. Given that the core layer is a generic isotropic material, the effect of laminate size on bistability is studied for various values of core modulus $(E)$ and thickness $(t)$. 


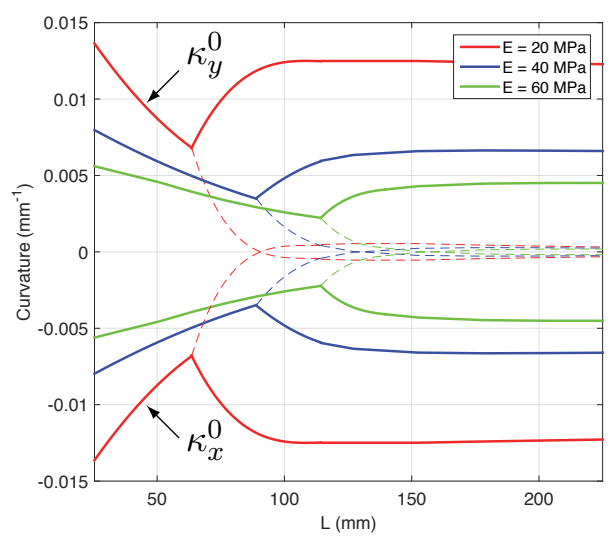

(a)

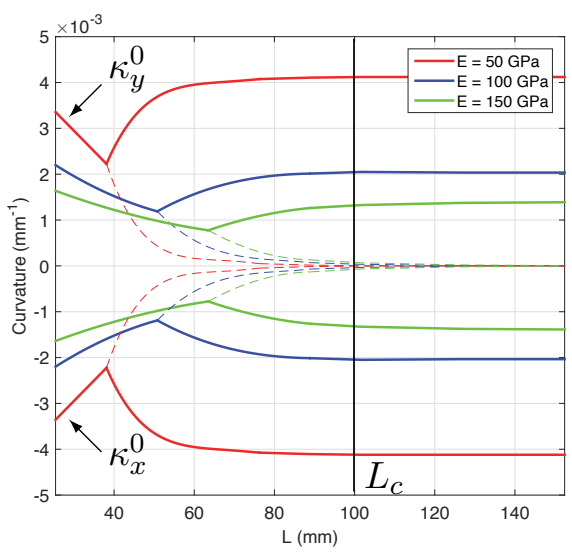

(b)

Figure 12: Effect of laminate size on bistability; (a)-(b) influence of core modulus on the critical characteristic length for bistability.

Figure 12(a) shows shape bifurcation plots for $E$ ranging from 20 - 60 $\mathrm{MPa}$ and $t=3 \mathrm{~mm}$. For a given $t, L_{b}$ and $L_{c}$ increase with an increase in core modulus. Beyond $L_{c}, \kappa_{x}^{0}$ and $\kappa_{y}^{0}$ have the same sign for each stable shape. For $E$ ranging from $50-100 \mathrm{GPa}$ at $t=0.254 \mathrm{~mm}$ (Figure 12(b)), $L_{b}$ is higher for higher $E$ whereas $L_{c}$ is independent of $E$. In this case, $\kappa_{x}^{0}$ and $\kappa_{y}^{0}$ have opposite signs beyond $L_{c}$.

From Figure 12, it is apparent that bifurcation length is a function of core modulus and thickness, and hence a function of the strain energy density of the core. With higher strain energy density, the bifurcation length of a laminate in a single shape (saddle) is higher. The critical characteristic length for cylindrical stable shapes is constant when the core modulus is on the order of $10 \mathrm{GPa}$ or higher. For a core whose modulus is higher than that of an elastomer (EMC) by atleast four orders of magnitude, the in-plane component of strain is negligible compared to its out-of-plane deflection component $((1)-(3)) . L_{c}$ is a result of the geometric nonlinearity associated with large out-of-plane deflection, and is constant in value beyond a particular core modulus. Although third-order polynomials are sufficient to describe the scaling effect in square laminates, higher order polynomials would be required to explain the loss in bistability in laminates with high aspect ratio [23]. 


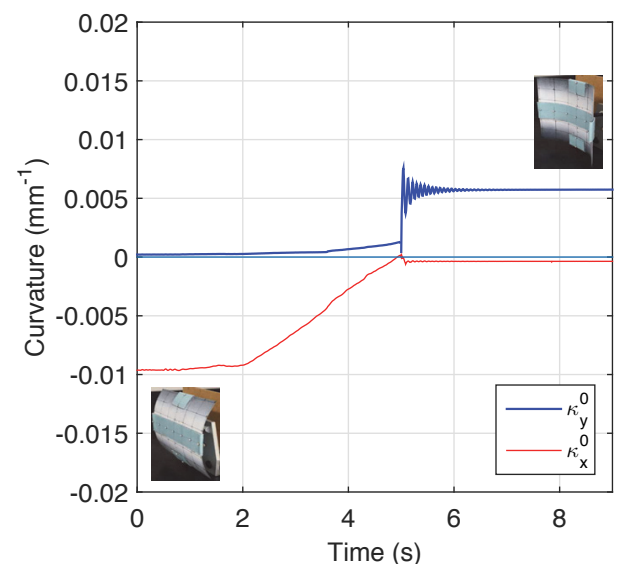

(a)

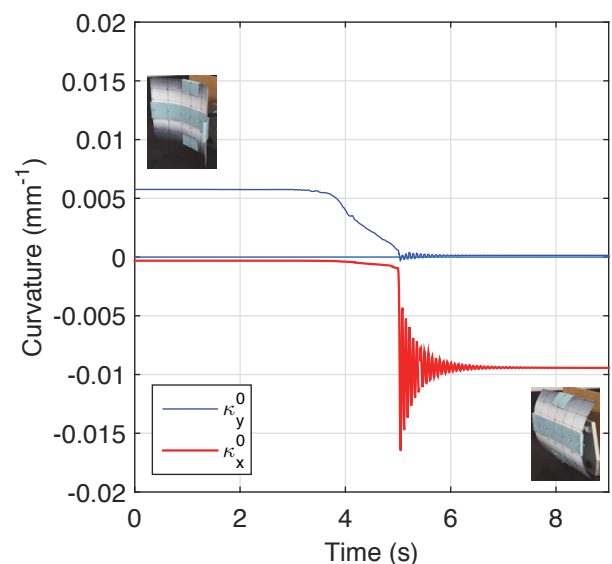

(b)

Figure 13: Measured dynamic response of a mechanically-prestressed bistable laminate during a transition from one stable shape to another.

\section{Response of the laminate to shape transition}

This section is a report on the measured dynamic response of a matrixprestressed bistable laminate during snap-through from one cylindrical shape to another. The motion capture camera setup described in Section 3.3 is used in this experiment. The tested sample is the same as the one shown in Figure $6(\mathrm{a})$. The sample is glued to the head of a thin bolt at its center to create a free boundary at the ends. The composite is initially curved about the $Y$ axis and a moment is manually applied to it about its horizontal axis to snap into the shape with curvature about the $X$ axis (Figure 13(a)). The laminate is then snapped back into its initial curvature and its response is recorded (Figure 13(b)). Ideally, the measured snap-through responses must be identical. However, the equilibrium curvatures of the fabricated sample are unequal (0.0057 and $0.0093 \mathrm{~mm}^{-1}$ ) and hence the measured responses are unequal. The vibratory amplitude is higher in the case where the laminate is snapped into the larger of the two curvatures. The frequencies corresponding to vibrations post the transitions shown in Figures 13(a) and 13(b) are measured to be 20 and $35 \mathrm{~Hz}$ respectively. 


\section{Concluding Remarks}

A room temperature-cured unsymmetric bistable laminate with tunable equilibrium shapes is proposed and demonstrated for the first time. A method for the fabrication of mechanically-prestressed bistable laminates is presented. An analytical model that includes the material and geometric nonlinearities of the laminae is developed to describe the mechanics of the composite. The simulated shapes of the laminate are in agreement with the measured shapes. The prescribed design methodology can be extended to develop bistable laminates with arbitrary EMC orientations. Mechanicallyprestressed bistable laminates offer possibilities for the design of adaptive structures for motion and vibration control through the incorporation of localized bistability in existing structures.

\section{Acknowledgements}

Financial support was provided by member organizations of the Smart Vehicle Concepts Center, a National Science Foundation Industry/University Cooperative Research Center (www.SmartVehicleCenter.org). Additional support for S.C. was provided by a Smart Vehicle Center Graduate Fellowship. Technical advice was provided by Dr. Umesh Gandhi and Mr. Kazuhiko Mochida from Toyota Technical Center (TEMA-TTC) in Ann Arbor, MI.

\section{References}

[1] Daynes S, Weaver PM. Review of shape-morphing automobile structures: concepts and outlook. Proc Inst Mech Eng D: J Automob Eng. 2013;227(11):1603-1622.

[2] Mattioni F, Weaver PM, Potter KD, Friswell MI. The application of thermally induced multistable composites to morphing aircraft structures. In: The 15th International Symposium on: Smart Structures and Materials \& Nondestructive Evaluation and Health Monitoring. International Society for Optics and Photonics; 2008. p. 693012-693012-11.

[3] Lachenal X, Daynes S, Weaver PM. Review of morphing concepts and materials for wind turbine blade applications. Wind Energy. 2013;16(2):283-307. 
[4] Kebadze E, Guest SD, Pellegrino S. Bistable prestressed shell structures. Int J Solids Struct. 2004;41(11-12):2801-2820.

[5] Seffen KA. Mechanical memory metal: a novel material for developing morphing engineering structures. Scr Mater. 2006;55(4):411-414.

[6] Norman AD, Seffen KA, Guest SD. Multistable corrugated shells. Proceedings of the Royal Society of London A: Mathematical, Physical and Engineering Sciences. 2008;464(2095):1653-1672.

[7] Brinkmeyer A, Santer M, Pirrera A, Weaver PM. Pseudo-bistable self-actuated domes for morphing applications. Int J Solids Struct. 2012;49(9):1077-1087.

[8] Eckstein E, Pirrera A, Weaver PM. Multi-mode morphing using initially curved composite plates. Compos Struct. 2014;109:240-245.

[9] Fernlund G, Rahman N, Courdji R, Bresslauer M, Poursartip A, Willden $\mathrm{K}$, et al. Experimental and numerical study of the effect of cure cycle, tool surface, geometry, and lay-up on the dimensional fidelity of autoclave-processed composite parts. Compos Part A Appl Sci Manuf. 2002;33(3):341-351.

[10] Hyer MW. Some observations on the cured shape of thin unsymmetric laminates. J Compos Mater. 1981;15(2):175-194.

[11] Hyer MW. Calculations of the room-temperature shapes of unsymmetric laminates. J Compos Mater. 1981;15:296-310.

[12] Schlecht M, Schulte K. Advanced Calculation of the Room-Temperature Shapes of Unsymmetric Laminates. J Compos Mater. 1999;33(16):14721490 .

[13] Lee H, Lee JG, Ryu J, Cho M. Twisted shape bi-stable structure of asymmetrically laminated CFRP composites. Compos Part B, doi: 101016/jcompositesb201609079. 2016;.

[14] Daynes S, Diaconu CG, Potter KD, Weaver PM. Bistable prestressed symmetric laminates. J Compos Mater. 2010;44(9):1119-1137.

[15] Daynes S, Potter KD, Weaver PM. Bistable prestressed buckled laminates. Compos Sci Technol. 2008;68(15-16):3431-3437. 
[16] Li H, Dai F, Weaver PM, Du S. Bistable hybrid symmetric laminates. Compos Struct. 2014;116:782-792.

[17] Etches J, Potter K, Weaver P, Bond I. Environmental effects on thermally induced multistability in unsymmetric composite laminates. Compos Part A Appl Sci Manuf. 2009;40(8):1240-1247.

[18] Bubert EA, Woods BKS, Lee K, Kothera CS, Wereley NM. Design and fabrication of a passive 1D morphing aircraft skin. J Intell Mater Syst Struct. 2010;21(17):1699-1717.

[19] Chillara VSC, Headings LM, Dapino MJ. Self-folding laminated composites for smart origami structures. In: ASME 2015 Conference on Smart Materials, Adaptive Structures and Intelligent Systems; 2015. p. 8968.

[20] Hyer MW. The room-temperature shapes of four-layer unsymmetric cross-ply laminates. J Compos Mater. 1982;16(4):318-340.

[21] Peel LD, Jensen DW. Nonlinear modeling of fiber-reinforced elastomers and the response of a "rubber muscle" actuator. Pap-Am Chem Soc: Div Rubber Chem. 2000;(30).

[22] Dano ML, Hyer MW. Thermally-induced deformation behavior of unsymmetric laminates. Int J Solids Struct. 1998;35(17):2101-2120.

[23] Pirrera A, Avitabile D, Weaver PM. Bistable plates for morphing structures: A refined analytical approach with high-order polynomials. Int J Solids Struct. 2010;47(25-26):3412-3425.

[24] Potter K, Weaver P, Seman AA, Shah S. Phenomena in the bifurcation of unsymmetric composite plates. Compos Part A Appl Sci Manuf. 2007;38(1):100-106.

[25] Hufenbach W, Gude M, Kroll L. Design of multistable composites for application in adaptive structures. Compos Sci Technol. 2002;62(16):22012207.

[26] Zhang Z, Wu H, He X, Wu H, Bao Y, Chai G. The bistable behaviors of carbon-fiber/epoxy anti-symmetric composite shells. Composites Part B. $2013 ; 47: 190-199$. 
[27] Daynes S, Weaver P. Analysis of unsymmetric CFRP-metal hybrid laminates for use in adaptive structures. Compos Part A Appl Sci Manuf. 2010 Nov;41(11):1712-1718.

[28] Reddy JN. In: Theory and analysis of elastic plates. Philadelphia, PA: Taylor \& Francis; 1999. p. 112.

[29] Chillara VSC, Headings LM, Dapino MJ. Multifunctional composites with intrinsic pressure actuation and prestress for morphing structures. Compos Struct. 2016;157:265-274.

[30] Galuppi L, Royer-Carfagni G. Shear coupling effects of the core in curved sandwich beams. Compos Part B. 2015;76:320-331.

[31] Foraboschi P. Three-layered sandwich plate: Exact mathematical model. Compos Part B. 2013;45(1):1601-1612.

[32] Betts DN, Salo AIT, Bowen CR, Kim HA. Characterisation and modelling of the cured shapes of arbitrary layup bistable composite laminates. Compos Struct. 2010;92(7):1694-1700.

[33] Murray G, Gandhi F, Bakis C. Flexible matrix composite skins for one-dimensional wing morphing. J Intell Mater Syst Struct. 2010;21(17):1771-1781. 